\title{
A Ferry Scheme for Geocast in MANETs
}

\author{
Ting Wang, Chor Ping Low \\ School of Electrical and Electronic Engineering, \\ Nanyang Technological University, Singapore
}

\begin{abstract}
In this paper, we propose a fully distributed scheme that uses a subset of nodes, namely ferries, to perform geographical broadcast tasks in a Mobile Ad Hoc Network (MANET). This scheme, referred to as the GeoFerry scheme, is to the best of our knowledge, one of the first scheme which adopts the deployment of ferries to address the geocast problem. Based on a theoretical analysis of the time intervals between the nodes' consecutive arrivals in a particular area in the network, we further improved the performance of the GeoFerry scheme by reducing the overall delay. Extensive simulations are carried out to validate the theory and the performance of our proposed scheme.
\end{abstract}

Index Terms-Mobile Ad Hoc Network, Geographical Multicast, Ferry Scheme, Inter-Arrival Time, Distributed Scheme, GeoFerry.

\section{INTRODUCTION}

A Mobile Ad Hoc Network (MANET), as described in [1], [2], is a kind of mobile wireless network, which is a collection of mobile hosts connected through wireless channels. The hosts of a MANET are usually called nodes while direct connections between the nodes are referred to as links. Nodes in a MANET send and receive packets to/from other nodes as terminals and forward the packets as intermediate routers at the same time. The routing path of a packet in a MANET is formed by a collection of mobile nodes. Packets are forwarded hop by hop. Since no infrastructure is required for MANETs, they are often used to provide temporary network services under emergency situations such as disaster relief assistance or area exploration.

In these applications, instead of sending information to a particular node, it is also desirable to send a message to a specific region in the network area. This type of network service is defined as Geographical Broadcast (Geocast). For example, when the rescue team need to check the situation in a building located inside the disaster area, they may not know which member of the team is near to the building at that time. The destination for a traditional network packet could not be determined. Geocast can be used in this situation to send the packet to the team members close to the building of interest. Although it was firstly introduced in [3] as a service in the traditional infrastructure networks, researchers also studied geocast in mobile sensor networks [4], vehicular network [5] and MANETs [6].

However, unlike the infrastructure network, the mobility of the nodes and the instability of the wireless links cause a highly dynamic topology in MANETs. When a link between two nodes breaks, one or several routing paths may be destroyed. This possibility of link breakage makes a critical strike on ad hoc routing because most protocols, including the geocast protocols, typically assume that the network is always connected. To restore connectivity and enhance the survivability of MANETs, many schemes are proposed. One of them, namely the Ferry Schemes [7], uses special nodes as ferries to deliver packets across disconnected parts of a MANET. While it is proven to be effective in many scenarios of MANETs applications, to the best of our knowledge, ferry schemes have not been used in geocast.

In this paper, we adopt the ferry scheme in geocast service and design a new scheme to solve the geocast problem, namely the GeoFerry Scheme. We will show that ferry (or ferries) can effectively shorten the delay of geocast and reduce the packet drop rate. Furthermore, we provide an adaptive scheme, based on the inter-arrival time of MANET nodes to a region inside the network area, to further reduce the overall delay in packet delivery. Section II of this paper introduces the application scenarios of geocast, while Section III gives a review on the existing works related to geocast and ferry schemes in MANETs. The GeoFerry scheme is discussed in Section IV. Our simulation and results are presented in Section V. Section VI concludes the paper.

\section{APPLICATION SCENARIOS}

MANETs are often used in area exploration and surveillance. In such applications, the tasks are closely related to the geographical area in the network, and less related to the identity of a particular node in the MANET. For example, when a MANET is used in a disaster relief scenario, the task of a node is usually to explore some area to find and locate survivors. In the task assignment, the location of the area is more important than which node goes to that area. In other words, we are more interested in "where" the node should move to than "which" node is going to complete the task. In such cases, new tasks can be treated as geocast requests or packets which need to be dispatched to a particular area (instead to a particular node).

Another scenario to use geocast is that when the destination node id is not known by the source node. In some large scale MANETs, such as [8], the nodes are not deployed at the same time. The id of of newly deployed nodes may not be known to the earlier deployed nodes. If an earlier deployed node (the source node) need to send message to a new node, of which the id is unknown, it could not use the normal communication protocol. However, if the source node knows where the destination node may be located, it could use geocast to first deliver the packet to an area that is closer to the destination node. Then, other nodes in this area may know 


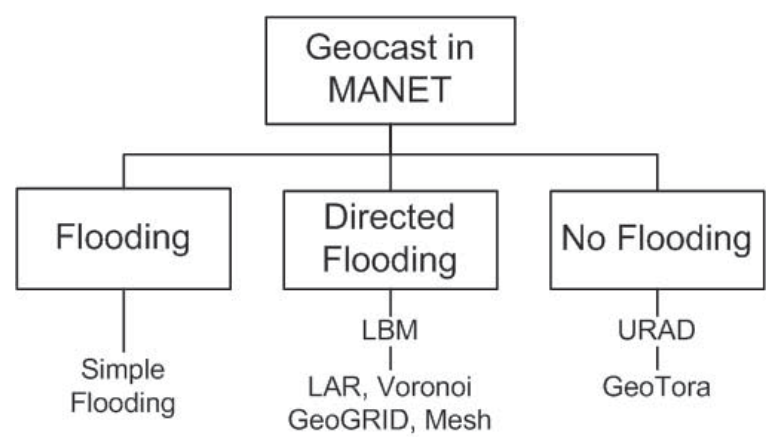

Fig. 1: Existing Geocast Schemes

the id of the newly deployed node (the destination node) and deliver the message.

\section{RELATED WORK}

\section{A. Geocast Problem}

Geocast [3] is defined as a service to deliver packets in a network to a specific geographic region, namely the destination region. The packets delivered by geocast are referred to as Geocast packets. In the destination fields of these packets, usually the coordinates (such as GPS coordinates) of the destination regions are stored instead of nod ids.

In some of the geocast implementations, the packets will be broadcasted to every host inside the destination regions, like in [9]. But in some other existing geocast protocols such as [6], [10], a geocast task is considered as completed when the packet is received by any one of the hosts inside the destination region. In this paper, we adopt the latter approach whereby the goal of GeoFerry scheme is to deliver a packet to at least one host in the destination region.

As summarized by Maihöfer in [6], the geocast schemes in MANETs can be classified as flooding, directed flooding and no flooding, as shown in Fig. 1. Although broadcasting the packets to all the hosts in the network could apparently solve the geocast problem, simple flooding is too costly as confirmed by [11]. Thus, we will focus our attention on the other two categories.

The directed flooding schemes are also referred to as Location Based Multicasting (LBM) schemes. Based on their locations, a subset of the nodes are selected as the forwarding nodes to deliver the geocast packets. In [12], Ko et al. proposed the Location-Aided Routing (LAR) scheme, in which a rectangular zone with the source node and the destination region on the diagonal line are defined as the forwarding zone of the geocast packet, as shown in Fig. 2a. The forwarding zones of LBM schemes are defined based on different criteria, such as the distance towards the destination region [13], or a voronoi graph generated from the network topology [10], [14]. These schemes assume there will be at least one node in the forwarding zone to receive and deliver the packets. This may not be true when the density of nodes in the MANET is low, which is a common phenomenon in the applications we described in Section I.
When flooding is not used, modified unicast protocols can be used to perform geocast in MANET. [15] provides a general discussion on these protocols, and referred them as Unicast Routing with Area Delivery (URAD) protocols. For example, GeoTORA [16], [13] is derived from the MANET routing protocol TORA [17]. Each packet will be forwarded on a path selected from the edges of a directional acyclic graph (DAG). However, these protocols inherit the weakness from the unicast protocols. For example, GeoTORA suffers from a long delay of constructing and updating the DAG. The DAG and forwarding path of the geocast packets are shown in Fig. 2b.

In both LBM and URAD, nodes movements may cause geocast delivery failure to occur. In Fig. 2a, if node $n_{1}$ moves to the shown direction, the link between $n_{1}$ and $n_{2}$ will break. This will result in the disconnection of the source node from all other nodes in the destination region, giving rise to two separation partitions of the network. Hence it will not be possible for geocast packets to be delivered from the source node to the destination region. In URAD, even when the disconnected parts can be detected by some protocols like TORA, but they are not able to actively restore the connection.

However, not all node movements are harmful to geocast. In Fig. $2 \mathrm{~b}$, node $n_{3}$ is moving towards the destination region. If other nodes are aware this movement and sends their packets to $n_{3}$, the geocast may be completed in a shorter time. This advantage of nodes movement has so far not utilized in the existing geocast schemes. GeoFerry aims to exploit active nodes movement to help deliver the geocast packets.

\section{B. Ferry Schemes}

The method of using message ferries is inspired from real life analog, where ferries are used to transport people across valleys or straits. In ferry schemes, a subset of nodes, referred to as ferry nodes (or ferries), is used to transmit information across the gaps between disconnected parts of the MANET. Other nodes which performs ordinary tasks are called normal nodes. One of the major challenges in the ferry schemes is to determine the desired destinations of ferry nodes, i.e. where the ferries should move to, to enhance packet delivery.

Many of the existing work such as [7] simply assumes that the normal nodes in the MANET are stationary or only have limited mobility. The construction of ferry routes can thus be optimized according to the locations of the nodes. However, mobile nodes is a common phenomenon in real life applications of MANETs. In [18], centralized control is used to determine the optimized location for ferries. Normal nodes need to update their locations and moving directions periodically. The communication cost is thus very high. In Ou et al.'s work [19], a first-in-first-out (FIFO) queue is built to store all possible locations for ferries. The ferry will then travel to the locations one by one until it finds a location which is not occupied by another ferry. This scheme is effective only when the number of ferries in the system is large. It also suffers from long delay in packet delivery. Prediction of the normal nodes location is used by Tariq et al. in [20]. However, this 


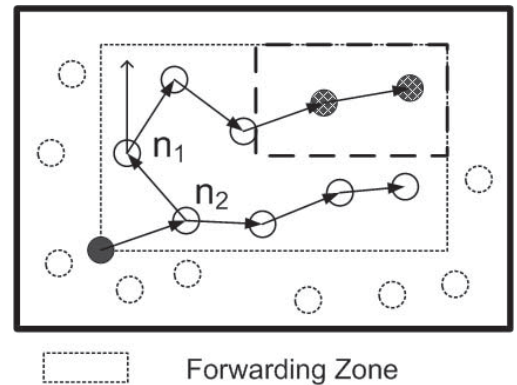

(a) LAR

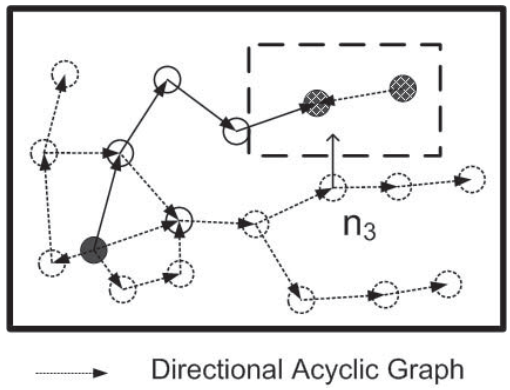

(b) GeoTORA

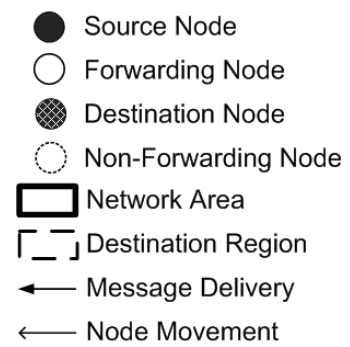

Fig. 2: Existing Geocast Schemes scheme is not distributed and not scalable with an increasing network area size.

In these existing ferry schemes, to approach the destination node of a packet, ferries need to be aware of the node's location. Updating the locations of the nodes causes long delay as well as much more communication efforts in the MANET. However, if we want to deliver a geocast packet, the destination will be available from the packet itself as we described in Section III-A, which makes the ferry scheme as a good candidate to solve the geocast problem.

\section{Design of the GeoFerry Scheme}

\section{A. System Model}

We assume a MANET consists of $n$ nodes in a given area $O$. There are $n_{N}$ normal nodes and $n_{F}$ ferries in the MANET, $n=n_{N}+n_{F}$. Each of the node is assigned with in ID $i, i \in$ $1,2, \ldots, n$ and it can be identified as $n_{i}$. Global positioning system (GPS) is equipped on both normal and ferry nodes. They are able to obtain their location information instantly. All the nodes are able to store the received packets in some storage, referred to as buffers.

Random Waypoint Mobility Model [21] is used to simulate the movement of the normal nodes. In this model, a node continuously selects a point, namely the waypoint, in the area $O$ and moves towards it. This is similar to the nodes movement when the MANET is used in area exploration or disaster relief assistance.

Initially, both normal nodes and ferries are uniformly distributed inside $O$. All the nodes select a waypoint and start to move with a speed uniformly distributed in the interval $\left[v_{\min }, v_{\max }\right]$, where $v_{\min }$ and $v_{\max }$ are the minimum and maximum speed of the nodes, respectively. Geocast packets will be generated by the normal nodes at random times. The node that generate packet $p$ is referred to as the source node of $p$. The destination region of $p$ will be a rectangular area inside $O$. The location and the size of the destination area are both randomly generated. In the applications we discussed in Section II, we can see the destination areas are usually much smaller than the network area. To simulate the real life applications, we restrict the size of the area to be smaller than one fourth of the size of $O$. The GPS information (in coordinate format) of the lower left corner of this rectangular area $\left(x_{d}, y_{d}\right)$, the length $l_{d}$ and the width $w_{d}^{1}$ of the area will be stored in the packet. Every node can locate the destination region based on these parameters when they receive the packet. The source node start to broadcast the packet using LBM as soon as it is generated. A packet will be dropped when it is timeout or the buffer of the receiving node is full.

\section{B. GeoFerry Scheme}

A ferry will be in either working or non-working states. In the working state, the ferry is helping to deliver some geocast packets, while in the non-working state, it moves according to the random waypoint model as the normal nodes. A boolean flag "WORK" is used to indicate which state the ferry is currently in. It is initialized as 0 , putting the ferry in the nonworking state.

The algorithms for the ferries in the GeoFerry scheme is showin in Table. I. In the algorithms, temp is a temporary data structure (such as the coordinates $(x, y)$ ) to represent a point in the network area. A first-in-first-out (FIFO) queue, namely wpQueue is used to store the waypoints.

Algorithm receive will be invoked when a packet (denoted as $p$ ) is received by the ferry. We assume the ferry receives $p$ from node $n_{p} . p$ will be stored in the ferry's buffer unless it is full. If the "WORK" flag is set to 1 , the ferry will add the center of the destination region of $p$ into wpQueue. The coordinate of the center can be computed as $\left(x_{d}+l_{d} / 2, y_{d}+w_{d} / 2\right)$. If the "WORK" flag is 0 , indicating the ferry is in non-working state, the ferry will set the "WORK" flag to 1 . The center of $p$ 's destination region will be set as the current waypoint. The ferry will inform node $n_{p}$ not to broadcast $p$ there after if $p$ is not dropped, and starts to move towards this new waypoint.

When the ferry reaches a waypoint, arrive algorithm will be invoked. The ferry will check the "WORK" flag again. If it is 1 , which means the current location is the center of the destination of some geocast packet the ferry has previously received, the node may try perform some tasks such as broadcasting or exploration, depending on the application

\footnotetext{
${ }^{1}$ Length is measured in $X$ direction of the coordinate system and width is measured in the $Y$ direction.
} 
TABLE I: Algorithms for Ferries in the GeoFerry Scheme

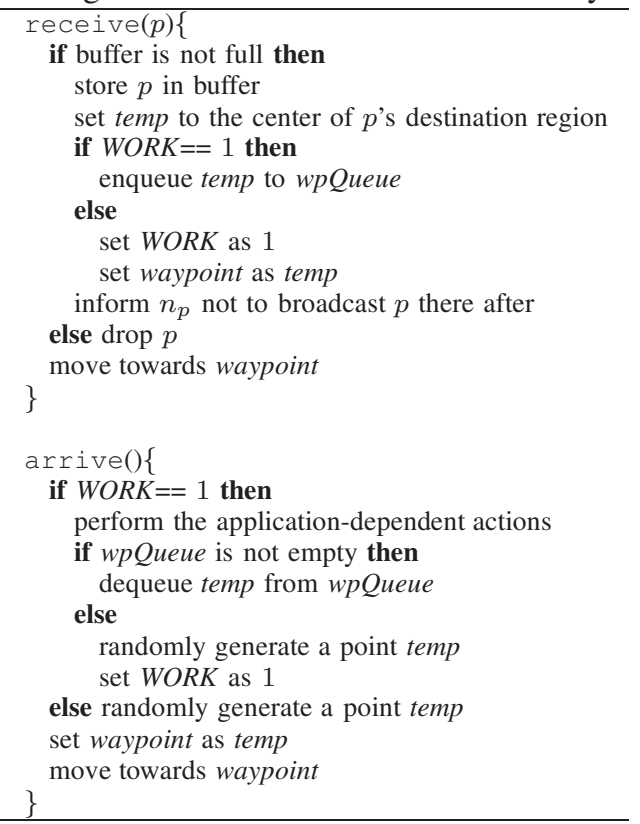

of this MANET. After that, it will dequeue a waypoint from the FIFO queue as the next destination. If the queue is empty, the "WORK" flag will be set back to 0, and the ferry will move towards a randomly generated waypoint. On the other hand, if "WORK" is 0 when it reaches the waypoint, the ferry will randomly generate a new waypoint and move towards it.

Intuitively, we could see that the active movement of ferries could deliver the geocast packets with shorter delay. In Section IV-C, an adaptive GeoFerry scheme is discussed.

\section{Adaptive GeoFerry Scheme}

The adaptive GeoFerry scheme is based on our observation and analysis on the inter-arrival times of the nodes for an area in a MANET. First, we focus on the inter-arrival time of a single node and then extend to the case of multiple nodes.

Assume a MANET has only one node namely node $n_{k}$. It moves continuously in $O$ according to the random waypoint mobility model. The inter-arrival time for $n_{k}$ for an area $A$ is defined as the time between node $n_{k}$ 's two consecutive arrivals in area $A$. Over a long period of time, node $n_{k}$ will arrive in area $A$ for multiple times. A series of inter-arrival time samples for this area can be obtained. We note that the statistical property of these inter-arrival times can reflect the frequency of a node arriving in the area, and the probability of a node arriving in that area during a time period. It is thus strongly related to our study of the geocast packet delivery.

We consider that a rectangular area $A$ as a potential destination region for some geocast packets. When $n_{k}$ moves towards a waypoint, it moves on a straight line. According to the convex property of a rectangular area, $n_{k}$ will arrive at area $A$ at most once, as it moves towards a waypoint. Since the waypoints are independently generated, the next arrival of $n_{k}$ in area $A$ is independent of the previous arrivals. Hence node $n_{k}$ 's arrivals in area $A$ are independent events.

Let $i_{T}$ denote the number of node $n_{k}$ 's arrivals in area $A$ during the time interval $(0, T)$. As node $n_{k}$ never stops moving, we have $i_{T} \rightarrow \infty$ as $T \rightarrow \infty$. We may assume that $i_{T}=\mu T, \mu$ will be the average rate per time unit of $n_{k}$ 's arrival in $A$.

We first consider one single arrival of node $n_{k}$ in area $A$ during the duration $T$. We define a smaller time duration $\left(t_{0}, t_{0}+\Delta\right)$. As a result of Bernoulli trials, the probability of the arrival taking place during $\left(t_{0}, t_{0}+\Delta\right)$ is $\Delta / T$.

Among all the $i_{T}$ arrivals, we use $r$ to denote the number of the arrivals which have taken place in the time duration $\left(t_{0}, t_{0}+\Delta\right)$. The probability $p_{i_{T}}(r, \Delta)$ of getting $r$ out of $i_{T}$ arrivals in a time interval $\Delta$ during the entire period $(0, T)$ can be estimated using the binomial probability mass function, where

$$
p_{i_{T}}(r, \Delta)=\frac{i_{T} !}{r !\left(i_{T}-r\right) !}\left(\frac{\Delta}{T}\right)^{r}\left(1-\frac{\Delta}{T}\right)^{i_{T}-r} .
$$

Note that $\Delta / T \rightarrow 0$ when $T \rightarrow \infty$. However,

$$
i_{T} \cdot \frac{\Delta}{T}=\mu T \cdot \frac{\Delta}{T}=\mu \Delta
$$

is a constant. Therefore, Eqn. 1 can be estimated using the Poisson theorem [22], where

$$
\frac{i_{T} !}{r !\left(i_{T}-r\right) !}\left(\frac{\Delta}{T}\right)^{r}\left(1-\frac{\Delta}{T}\right)^{i_{T}-r} \underset{i_{T} \rightarrow \infty}{\rightarrow} e^{-\mu \Delta} \frac{(\mu \Delta)^{r}}{r !} .
$$

The inter-arrival time $X$ is greater than $t$ if there is no arrivals in time interval $\left(t_{0}, t_{0}+t\right)$. From Eqn. 1 and 3, we have

$$
p(X>t)=p_{i_{T}}(0, t)=e^{-\mu t} .
$$

Therefore $X$ is exponentially distributed with expected value equals to

$$
E[X]=1 / \mu .
$$

This also shows that the arrivals of node $n_{k}$ in area $A$ is a Poisson process.

However, usually we have multiple identical nodes in a MANET. We re-define the inter-arrival time in a multiple nodes case as the time interval between two arrivals in the same area by any node(s). In this condition, the arrivals for each node in an area are independent and identical Poisson processes. According to the superposition property of Poisson process, if $n$ independent identical Poisson processes overlap, the resultant process will also be a Poisson process and the mean inter-arrival time becomes $1 / n$ of the original mean inter-arrival time in a single process.

In our scenario, if we use $X_{n}$ to denote the inter-arrival time for an area where $n$ is the number of nodes in the MANET, we will have

$$
E\left[X_{n}\right]=1 /(\mu n)=E\left[X_{1}\right] / n .
$$

Simulations are carried out to validate our theoretical analysis. We measure the inter-arrival times for four different areas $A_{1}, A_{2}, A_{2}^{\prime}$ and $A_{3}$ inside a square area as shown in Fig. 3. 


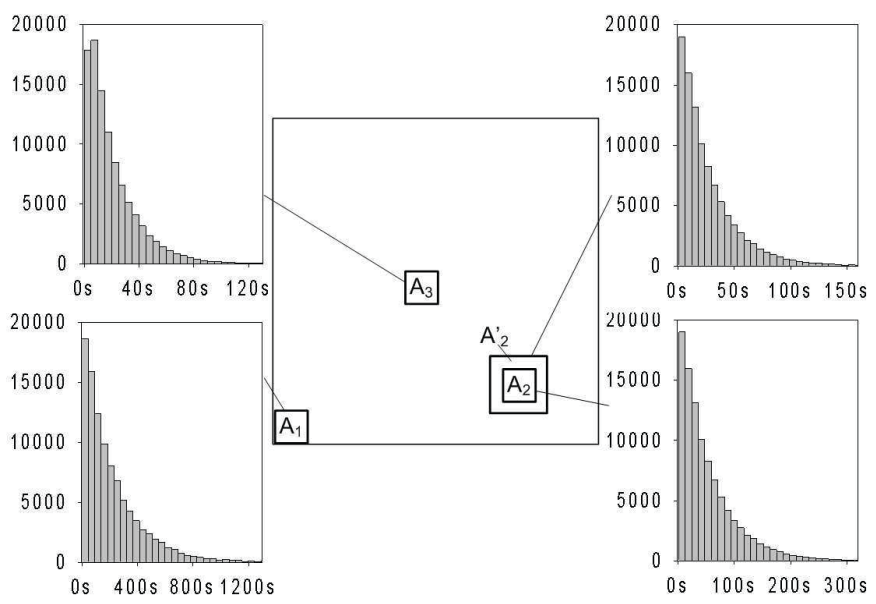

Fig. 3: Inter-Arrival Times for Different Areas

$A_{1}$ has a the same size as $A_{2}$ and $A_{3} . A_{2}^{\prime}$ 's size is twice of $A_{2}$ but centered at the same location as $A_{2}$. There are 5 nodes in the MANET and 100000 samples of the inter-arrival time are taken for each area.

The histograms that depict the inter-arrival time for the corresponding areas are also shown in Fig. 3. In each histogram, the inter-arrival times are equally divided into 50 nonoverlapping categories, shown on the $x$-axis. Each category represents a range of time length ${ }^{2}$. For example, each category of the histogram which corresponds to area $A_{3}$ has a time interval of $120 \mathrm{~s} / 50=2.4 \mathrm{~s}$. Each bar along the $y$-axis is used to show the number of inter-arrival times that fall in each category. We can see that the inter-arrival times for all the four areas follow an exponential distribution but with different means. This can also be confirmed by a very good fitting of the curves to the exponential distribution probability density function. The fitting results were also shown in [23].

From the above analysis we can see, when the number of nodes in the MANET is fixed, the expected value of the interarrival time, $E\left[X_{n}\right]=1 /(\mu n)$ depends on the factor $\mu$. From in Fig. 3, we can conclude that $\mu$ depends on the location and the size of the rea $^{3}$.

When an area is closer to the center of $O$, such as area $A_{3}$, the inter-arrival time will be shorter than those areas closer to the edge of $O$ (such as $A_{2}$ ). The closer to the edge of $O$, the longer the inter-arrival times are (comparing $A_{2}$ with $A_{1}$ ). This is because of the well-known border effect of random waypoint mobility model in which nodes tend to cross the center area more often than the borders. We can also see that a larger area will have a shorter inter-arrival time than a smaller area which are both centered at the same location (comparing $A_{2}^{\prime}$ to $A_{2}$ ).

In geocast applications, if the destination area of a packet $p_{1}$ has a smaller inter-arrival time than another packet $p_{2}$, it

\footnotetext{
${ }^{2}$ This length of time is different in different histograms.

${ }^{3} \mathrm{We}$ also note that $\mu$ is related to the speed of the node, but it is less relevant to our discussion here.
}

TABLE II: Adaptive Algorithm

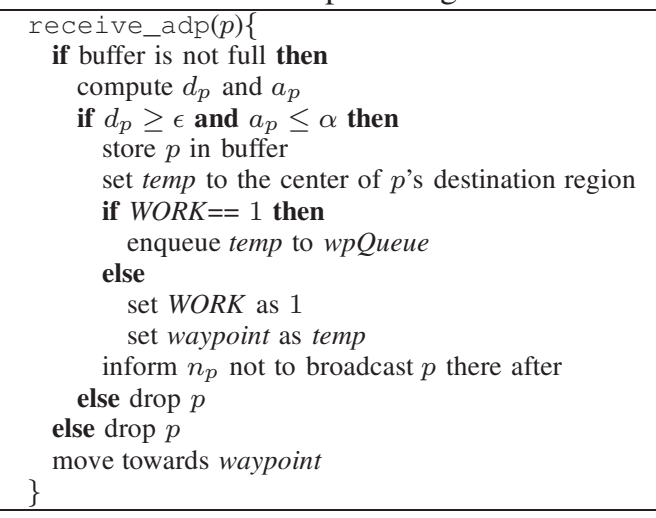

means normal nodes have a higher probability to arrive in $p_{1}$ 's destination area and deliver $p_{1}$, i.e. $p_{2}$ is more likely to be dropped before being delivered. Hence in the design of the GeoFerry scheme, the ferries should accord higher priority to deliver $p_{2}$ as compared to $p_{1}$. In this way, ferries can choose which packets to deliver based on the inter-arrival time of the destination area of the packets. With this selection, the ferry do not have to move to all the centers of the destination areas of the packets it received. Time and energy can thus be saved, which in turn helps to improve the performance of GeoFerry scheme.

To compute the exact inter-arrival time for some area, the ferry node needs to know the total number of normal nodes in the MANET and their speed. However, because we want GeoFerry to be a fully distributed scheme, we will estimate the inter-arrival time by using two parameters $\alpha$ and $\epsilon$.

When a packet $p$ is received by a ferry, the ferry will measure the distance between the center of the network area and the center of destination region of certain packet $p$, denoted as $d_{p}$, and the size of the destination area, denoted as $a_{p}$. If $d_{p}<\epsilon$ or $a_{p}>\alpha$, which means the destination area of $p$ has a small inter-arrival time, the ferry will not change its path of moving for $p$. Only when $d_{p} \geq \epsilon$ and $a_{p} \leq \alpha$, which means that is it unlikely that normal nodes can reach the destination area in a short time duration, ferry will store the center of its destination region into the FIFO queue and move towards it in due course. Table. II shows the pseudo code for the adaptive receive algorithm, namely receive_adp. We will show this optimization strategy effectively shorten the delivery delay in GeoFerry scheme while the delivery rate is not compromised.

\section{Simulation Results}

In the simulations, we used a $100 \mathrm{~m} \times 100 \mathrm{~m}$ area as $O$. Node speed is uniformly generated in the interval $[9 \mathrm{~m} / \mathrm{s}, 11 \mathrm{~m} / \mathrm{s}]$. The size of the geocast packets is $100 \mathrm{~kb}$ and the transmission rate is $1 \mathrm{Mb} / \mathrm{s}$. The timeout constant is set to $1000 \mathrm{~s}$. The generation rate of geocast packets is $0.05(\mathrm{pkt} / \mathrm{sec})$. The buffer size for all the nodes is $1 \mathrm{Mb}$ (i.e. 10 geocast packets). The maximum distance between two nodes to achieve successful communication is $5 \mathrm{~m} .100000$ geocast packets are generated 
in every simulation, and repeated for different values of $n_{N}$ and $n_{F}$. We increased $n_{N}$ from 5 to 50 with a step size of 5. For each $n_{N}$ value, $n_{F}$ is varied from 1 to 5 . As benchmarks, LBM and URAD 4 are also simulated. They cover the directed flooding and no flooding categories of existing MANET geocast schemes we discussed in Sect. III. In fact, LBM is the case when $n_{F}=0$.

The comparison between GeoFerry and LBM, URAD is shown in Fig. 4. In Fig. 5, the performance of GeoFerry with different number of ferries $\left(n_{F}\right)$ is shown. In Fig. 6, we show that when we use single ferry, and set $\epsilon=20, \alpha=10$, the adaptive GeoFerry scheme can further improve the geocast performance as described in Section IV-C.

All the existing simulation tools such as $n s-2$ or QualNet do not support a node to change its moving direction and speed according to a packets received (the movement of a node is recorded before the simulation of network traffic starts). Therefore we have to build up our own c++ programs in the simulation. To simulate LBM and URAD, we followed the original papers as closely as possible. The result are similar to other existing works such as [6].

\section{A. Failure Ratio}

Some of the geocast packets may fail to be delivered to the destination region before it is timeout or dropped. We divide the number of these failed packets by the total number of generated geocast packets to obtain a failure ratio of each scheme in the simulation.

In Fig. 4a, the failure ratios with different schemes shown. URAD has the highest failure rate. This is because the unicast protocols do not guarantee delivery of the packets especially when the topology of the network is not stable. The failure ratio of LBM decreases when $n_{N}$ increases. Because LBM uses broadcast to deliver the packets, when the density of the nodes are high, it becomes less likely that the packet cannot be delivered. When GeoFerry scheme is used, the failure ratio is decreased to below 0.05 even when there is only 1 ferry in the MANET.

As shown in Fig. 5a, the failure ration decreases further when we add more ferries in the MANET. With more ferries in the network, each ferry will get less packets to deliver. Thus the wpQueue becomes shorter for each ferry, and the packets can be delivered earlier. However, this result is less significant when the number of nodes is large. This is because when the node density is high, the failure ratio is already very small. Hence it becomes more difficult to show the improvements.

The failure ratio of the adaptive GeoFerry scheme is smaller than the original scheme (as shown in Fig. 6a). This is because when some geocast packets are discarded by the ferry, the ferry can deliver others in a shorter time. This avoid the packets to be dropped due to buffer overflow and timeout. This results validates the effectiveness of the adaptive scheme in packet delivery.

\footnotetext{
${ }^{4} \mathrm{LBM}$ uses rectangular forwarding zone and URAD uses AODV as the unicast protocol.
}

\section{B. Average Delay}

Delay is the time spend to transmit the geocast packets to the destination region. It is measured from the moment the packet is generated till the first node (either normal node of ferries) carrying the packet reaches the destination region. We measure the average delay of all delivered geocast packets. We can see form Fig. 4b, URAD has the highest average delay for all the values of $n$. Delay in LBM is less than URAD but higher than GeoFerry scheme. The performance of geocast is improved by GeoFerry scheme.

When number of ferries increases, delay in GeoFerry schemes decreases, as plotted in Fig. 5b. This shows the ferries can effectively help the MANET to deliver geocast packets with shorter delay, thus meeting the intended objective of this scheme.

The delay can be further decreased in the adaptive GeoFerry scheme, as shown in Fig. 6b. When only the packets with destination region on the edge of the area are delivered by the ferries, less distance may be traveled by the ferries. Less time will be spent before the packet arrives in the destination region.

\section{Ferry Delivery Ratio}

A well known result in MANETs is that the delay and failure rate will reduce when there are more normal nodes in the network. We want to show that the results in Section V-A and $\mathrm{V}-\mathrm{B}$ are not due to the increased total number of nodes. In order to do this, we measure ratio of packets delivered by ferries among all the delivered packets, as the Ferry Delivery Ratio. A high ferry delivery ratio would imply that it is the ferries which have enhanced the delivery of geocast packets, not the normal nodes. The results are shown in Fig. 5c and $6 c$.

As no ferry is used in LBM and URAD, the ratios for these two schemes are always 0 . In the GeoFerry scheme, at least about $20 \%$ of the packets are delivered by ferries. This ratio increases as the number of ferries increases. When there are 5 ferries in the network, about half of the packets are delivered by ferries. This shows the ferries are actively enhancing the geocast in this network. The ratio also drops slightly when $n_{N}$ increases, because when there are more normal nodes, they can deliver the packets using LBM before ferry arrives in the destination region.

In Fig. 6c, less packets are delivered by ferries in the adaptive scheme. But together with Fig. 6a and Fig. 6b, it can be shown that although some of the packets are left to the normal nodes to transmit, the effectiveness of GeoFerry scheme is not compromised.

\section{Fairness}

To further validate the correctness of the GeoFerry scheme, we show that using GeoFerry, the delivery of geocast packets are shared fairly among the nodes. The same simulation scenario as above sections is used to measure the number of packets delivered by each node. 


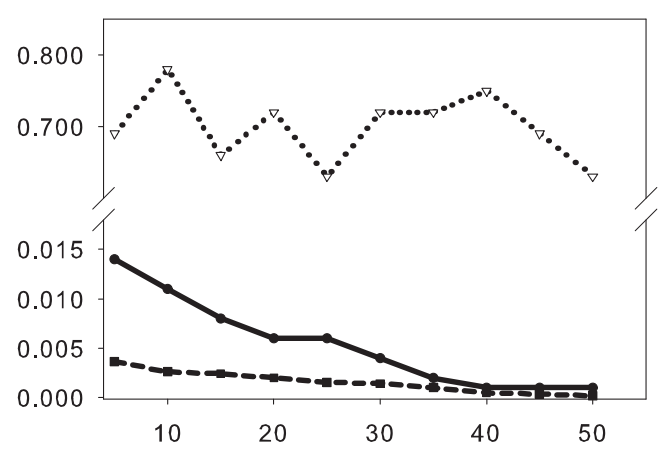

(a) Failure Ratio

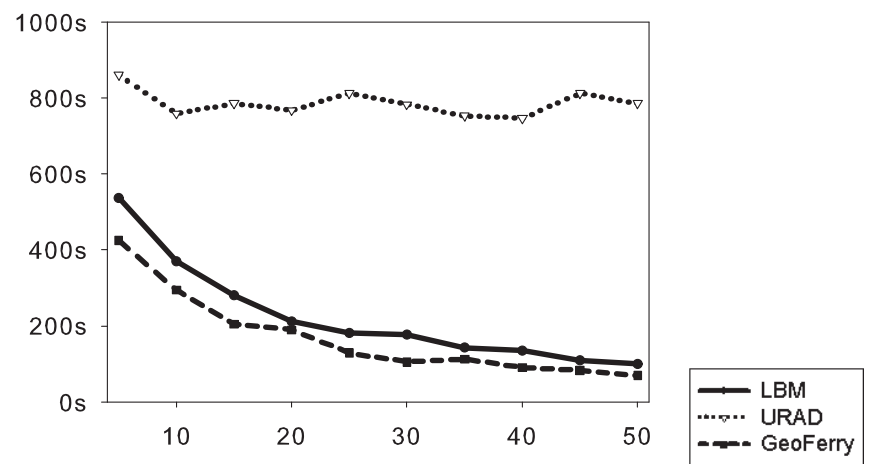

(b) Average Delay

Fig. 4: Simulation Results with Single Ferry

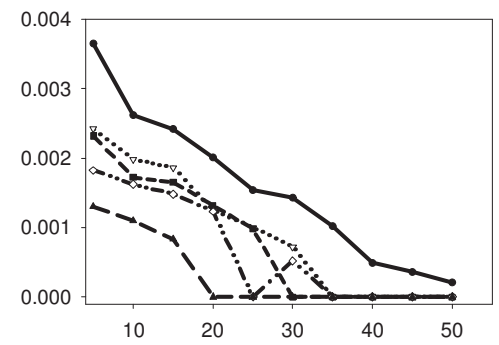

(a) Failure Ratio

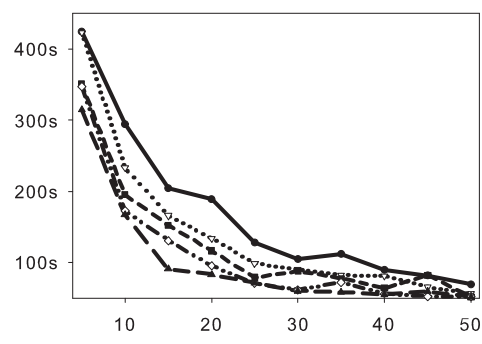

(b) Average Delay

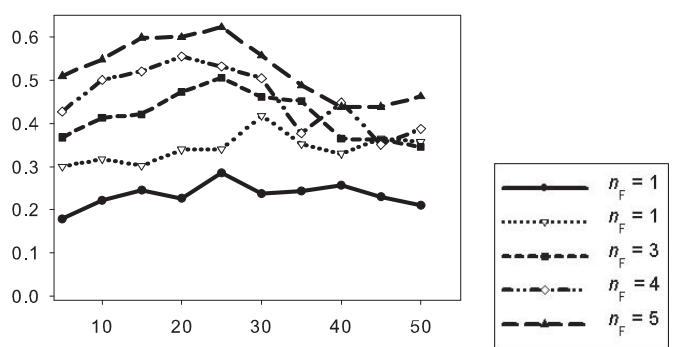

(c) Ferry Delivery Ratio

Fig. 5: Simulation Results with Multiple Ferry

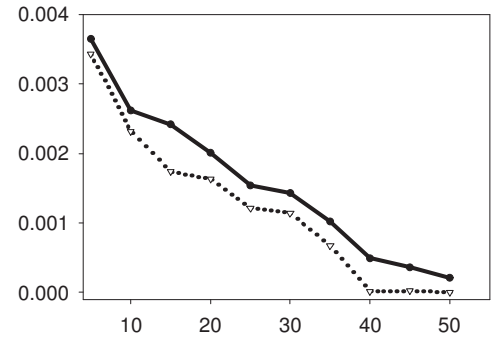

(a) Failure Ratio

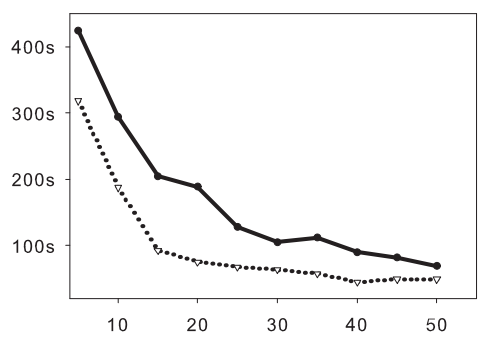

(b) Average Delay

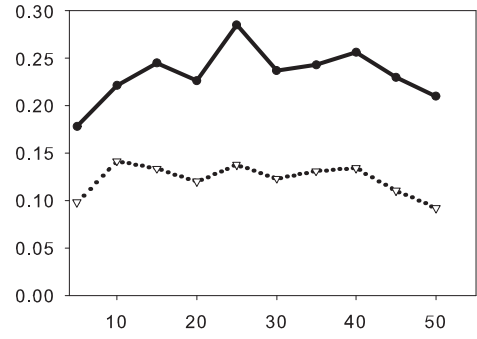

(c) Ferry Delivery Ratio

Fig. 6: Simulation Results with Adaptive GeoFerry Scheme

In Fig. 7, the $x$ axis is the ID of node. In Fig. 7a, nodes with ID from 1 to 10 are the ten normal nodes, and node 11 is the ferry. The undelivered packets are shown as with ID -1 . In Fig. 7 b, nodes 1 to 40 are normal nodes, nodes 41 to 43 are ferries. The length of each bar represents the number of geocast packets delivered by the corresponding node. From these figures we can see the packets are shared fairly among the normal nodes and the ferries are being used effectively for packet delivery.

\section{CONCLUSION}

In this paper, we propose a new scheme, namely the GeoFerry scheme, as a fully distributed solution to geocast in MANET. To the best of our knowledge, GeoFerry is one of the first schemes which adopt the use of ferries in geocast. Ferries are used to deliver the geocast packets directly to the destination region without repeatedly broadcasting the packets to its neighbors. The delay is thus reduced, and the delivery rate is also enhanced. Simulation shows that GeoFerry can effectively enhance the delivery of the geocast packets.

We further improve the scheme by making it adaptive to 


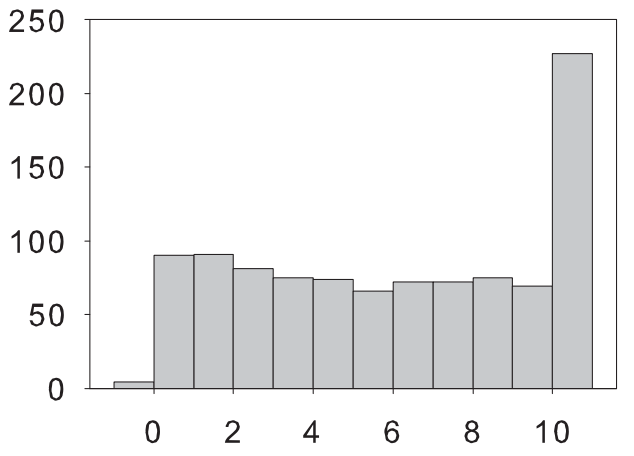

(a) $n_{N}=10, n_{F}=1$

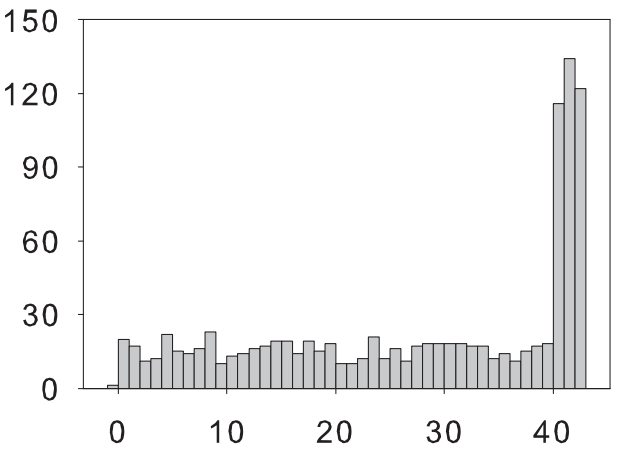

(b) $n_{N}=40, n_{F}=3$

Fig. 7: Fairness

the inter-arrival time of nodes (which moves according to the random waypoint model) in an area. We theoretically prove of that the arrivals of the nodes is a Poisson process and the interarrival time is exponentially distributed. This result is also validated by our simulation results. Based on these studies, we conclude that the expected value of the inter-arrival time of a node in an area depends on the location and the size of the area. Using this result, we devise an adaptive GeoFerry scheme which is able to reduce the average delay without compromising on the packet delivery ratio.

\section{REFERENCES}

[1] S. Corson, Mobile Ad hoc Networking (MANET): Routing Protocol Performance Issues and Evaluation Considerations (RFC 2501), Network Working Group, IEFT, January 1999.

[2] C. E. Perkins, Ad Hoc Networking. Addison-Wesley, 2001.

[3] G. Finn, "Routing and Addressing Problems in Large Metropolitan-Scale Internetworks," University of Southern California, ISI Research Report ISI/RR-87-180, March 1987.

[4] T. He, J. A. Stankovic, C. Lu, and T. F. Abdelzaher, "A Spatiotemporal Communication Protocol for Wireless Sensor Networks," IEEE Transactions on Parallel and Distributed Systems, vol. 16, no. 10, pp. 995-1006, 2005.

[5] A. Bachir and A. Benslimane, "A Multicast Protocol in Ad hoc Networks Inter-Vehicles Geocast," The 57th IEEE Semiannual Vehic. Tech. Conf. (VTC2003-Spring), vol. 4, pp. 2456 - 2460, April 2003.

[6] C. Maihöfer, "A Survey on Geocast Routing Protocols," IEEE Coтmunications Surveys and Tutorials, vol. 6, no. 2, pp. 32-42, 2004.

[7] W. Zhao and M. H. Ammar, "Message Ferrying: Proactive Routing in Highly-Partitioned Wireless Ad Hoc Networks," in Proceedings of the The Ninth IEEE Workshop on Future Trends of Distributed Computing Systems (FTDCS'03). Washington, DC, USA: IEEE Computer Society, 2003, p. 308.

[8] R. C. Shah, S. Roy, S. Jain, and W. Brunette, "Data mules: Modeling a three-tier architecture for sparse sensor networks," in Proc. of the First IEEE International Workshop on Sensor Network Protocols and Applications, 2003, pp. 30-41.

[9] T. Imielínski and J. C. Navas, "GPS-Based Geographic Addressing, Routing, and Resource Discovery," Communications of the ACM, vol. 42, pp. 86-92, 1999.

[10] C.-Y. Chang, C.-T. Chang, and S.-C. Tu, "Obstacle-Free Geocasting Protocols for Single/Multi-Destination Short Message Services in Ad Hoc Networks," Wirel. Netw., vol. 9, no. 2, pp. 143-155, 2003.

[11] Y.-C. Tseng, S.-Y. Ni, Y.-S. Chen, and J.-P. Sheu, "The Broadcast Storm Problem in a Mobile Ad Hoc Network," Wirel. Netw., vol. 8, no. 2/3, pp. 153-167, 2002.

[12] Y.-B. Ko and N. H. Vaidya, "Location-Aided Routing (LAR) in Mobile Ad Hoc Networks," Wirel. Netw., vol. 6, no. 4, pp. 307-321, 2000
[13] — "Anycasting-Based Protocol for Geocast Service in Mobile Ad Hoc Networks," Comput. Netw., vol. 41, no. 6, pp. 743-760, 2003.

[14] I. Stojmenovic, A. P. Ruhil, and D. K. Lobiyal, "Voronoi Diagram and Convex Hull Based Geocasting and Routing in Wireless Networks: Research Articles," Wirel. Commun. Mob. Comput., vol. 6, no. 2, pp. 247-258, 2006.

[15] C. Maihöfer, W. Franz, and R. Eberhardt, "Stored Geocast," in Proceedings of Kommunikation in Verteilten Systemen (KiVS. Springer Verlag, 2003, pp. 257-268.

[16] Y.-B. Ko and N. H. Vaidya, "GeoTORA: A Protocol for Geocasting in Mobile Ad Hoc Networks," in Proceedings of the 2000 International Conference on Network Protocols (ICNP '00). Washington, DC, USA: IEEE Computer Society, 2000, p. 240.

[17] V. D. Park and M. S. Corson, "A Highly Adaptive Distributed Routing Algorithm for Mobile Wireless Networks," in Proceedings of the Sixteenth Annual IEEE Conference on Communications Societies (INFOCOM '97). Washington, DC, USA: IEEE Computer Society, 1997, p. 1405.

[18] K.-F. Ssu, C.-H. Chou, W.-T. Wang, and S.-H. Chung, "Improving Data Transmission with Helping Nodes for Geographical Ad Hoc Routing," Computer Network, vol. 51, no. 18, pp. 4997-5010, 2007.

[19] C.-H. Ou, K.-F. Ssu, and H. C. Jiau, "Connecting Network Partitions with Location-Assisted Forwarding Nodes in Mobile Ad Hoc Environments," in Proceedings of the 10th IEEE Pacific Rim International Symposium on Dependable Computing (PRDC'04). Washington, DC, USA: IEEE Computer Society, 2004, pp. 239-247.

[20] M. M. B. Tariq, M. Ammar, and E. Zegura, "Message Ferry Route Design for Sparse Ad Hoc Networks with Mobile Nodes," in Proceedings of the 7th ACM international symposium on Mobile Ad Hoc Networking and Computing (MobiHoc '06). New York, NY, USA: ACM, 2006, pp. 37-48.

[21] J. Broch, D. A. Maltz, D. B. Johnson, Y.-C. Hu, and J. Jetcheva, "A Performance Comparison of Multi-Hop Wireless Ad Hoc Network Routing Protocols," in Proc. of the 4th Ann. ACM/IEEE Int. Conf. Mobile Computing and Netw., 1998, pp. 85-97.

[22] A. Papoulis and U. S. Pillai, Probability, Random Variables and Stochastic Processes with Errata Sheet. McGraw-Hill Science/Engineering/Math, December 2001.

[23] M. M. B. Tariq, "Interarrival Times for the Random Waypoint Model are Exponentially Distributed," http://www.cc.gatech.edu/ mtariq/pub/notes/rwpinterarrivals.pdf. 